(C) IEEE. Personal use of this material is permitted. However, permission to reprint/republish this material for advertising or promotional purposes or for creating new collective works for resale or redistribution to servers or lists, or to reuse any copyrighted component of this work in other works must be obtained from the IEEE.

This material is presented to ensure timely dissemination of scholarly and technical work. Copyright and all rights therein are retained by authors or by other copyright holders. All persons copying this information are expected to adhere to the terms and constraints invoked by each author's copyright. In most cases, these works may not be reposted without the explicit permission of the copyright holder. 


\title{
ADAPTIVE OBJECT-BASED IMAGE COMPRESSION USING WAVELET PACKETS
}

\author{
Dominik Engel and Andreas Uhl \\ Department of Scientific Computing, University of Salzburg \\ \{dengel,uhl\}@cosy.sbg.ac.at
}

\begin{abstract}
In this work we discuss an object-based wavelet image coding system which employs the wavelet packet best basis algorithm to adapt to each object separately. In addition to the increased functionality inherent to any object-based codec, the enhanced rate-distortion performance for images exhibiting objects with different frequency properties is shown.
\end{abstract}

Keywords: Wavelets, wavelet packets, adaptive image compression, object-based coding.

\section{INTRODUCTION}

With the finalization of the wavelet based JPEG2000 standard and the inclusion of a wavelet algorithm for synthetic/natural hybrid coding in MPEG-4 there is no doubt left that wavelet compression has to be considered state of the art nowadays.

With the emerge of MPEG-4, object-based compression techniques facilitating user interaction and many other features attracted the interest of the coding community. In this paper we describe an object-based wavelet image codec based on the wavelet packet best basis algorithm [1], which performs an adaptive optimization of the frequency resolution of the overcomplete wavelet packet basis. The idea is to adapt to different objects separately thereby achieving superior rate-distortion performance as compared to global adaptation. Therefore, we result in two advantages of the object-based approach: better functionality and better compression results.

\section{OBJECT-BASED COMPRESSION USING WAVELET PACKETS}

Wavelet packets [2] represent a generalization of the method of multiresolution decomposition and comprise the entire family of subband coded (tree) decompositions. Whereas in the wavelet case the decomposition is applied recursively to the coarse scale approximations only (leading to the well known (pyramidal) wavelet decomposition tree), in the wavelet packet (WP) decomposition the recursive procedure is applied to all the coarse scale approximations and detail signals, which leads to a complete WP tree. The best basis algorithm selects the most suitable frequency subbands for signal compression by optimizing additive information cost functions in a rate-independent way. Recently, WP based compression methods have been developed which outperform the most advanced wavelet coders significantly for textured images in terms of rate-distortion (R-D) performance (e.g. for fingerprints and the famous testimage "Barbara" [3]). Finally the "double tree" and "T-F-tree" algorithms calculate adaptive spatial and frequency tilings jointly and therefore generalize the best basis algorithm even more [4].

Although from a R-D point of view there are better methods [5] than the best-basis algorithm, we have chosen to employ this method due to its relatively low computational complexity. In the following, we apply the best basis algorithm in an object-based environment, in which the aim is to construct WP bases fitted for each object in the image separately. 
Besides the increase in functionality, the obvious additional advantages of an object-based approach are the possibility to adapt the wavelet packet subband structure to each object and thus achieve better overall rate-distortion performance, and the possibility to grant different bitrates to objects, according to their importance in the image and/or their visual activity level. We assume the image to be already segmented into objects. The proposed compression algorithm is divided into three stages (Figure 1):

1. For each object, the best-basis algorithm is used to find the best-adapted basis for that particular object and transforms the pixels contained in it. During the analysis-step, the object borders have to receive special treatment to extend the signal [6]. If biorthogonal filters are used, the treatment of object borders is not limited to periodical extension, but may also use different kinds of symmetric extensions.

The structure of the best suited subband-decomposition is written to the final bitstream as a quad-tree for each object.

2. We then use an algorithm similar to SPIHT to exploit the hierarchical structure of the coefficients in the wavelet packet tree [7] (SMAWZ). SMAWZ uses the foundations of SPIHT, most importantly the zero-tree paradigm, and adapts them to wavelet packets. Whereas full wavelet packet decomposition is of order $O(N \log N)$ in contrast to $O(N)$ for pyramidal decomposition, the pure coding time SMAWZ takes for operation, like SPIHT, scales linearly with the number of output-bits.

SMAWZ can work in two modes, simple and improved.

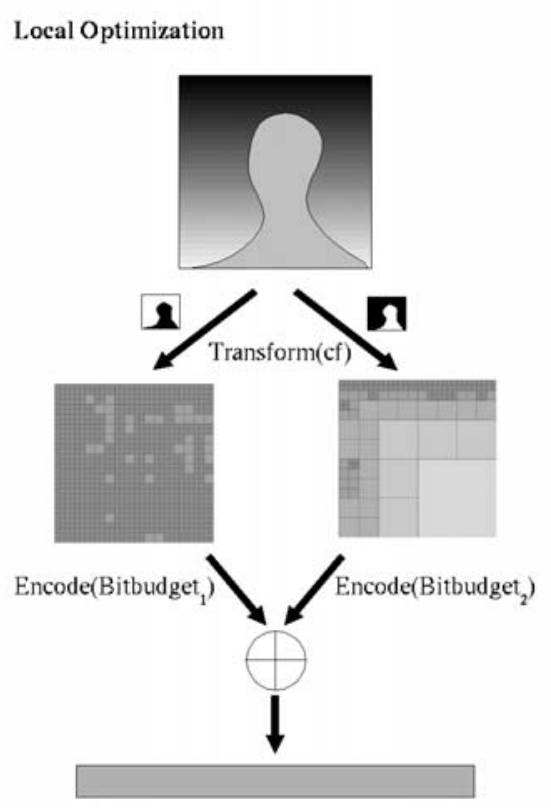

Fig. 1 Object oriented optimization. While the simple mode produces the same bits as SPIHT, the order of the bits is different. It is shown in [8] that for optimized PSNR at any rate, bigger coefficients have to come first in the bitstream. Thus, in the improved version SMAWZ takes care that unimportant bits are evaluated as late as possible. As the increase in computational complexity is rewarded by a significant gain in PSNR-performance, our testruns all use the improved mode of SMAWZ.

The encoding procedure is stopped at the bitrate assigned to the current object. The output is then written to the final bitstream. In this stage, different algorithmic variations are possible, for example to make the coefficients related to a specific object appear first in the bitstream making it possible to grant certain objects priority in progressive decoding.

3. Finally, the resulting bitstream, containing the object mask, the structure of the wavelet packet tree and the wavelet coefficients for each object is processed by an arithmetic encoder.

\section{EXPERIMENTAL RESULTS}

In our experiments, the above approach was implemented using the SMAWZ codec with the classical biorthogonal (7,9) filter. The tested images (Figure 2) all exhibit high frequency texture. The costfunctions employed are the Logarithm of Energy ( $\log E)$, the $\ell$-Norm and the Entropy Information Cost (EIC) [2], which are all additive costfunctions, meaning that 
for a set of subbands $s_{1}, \ldots, s_{n}$ and a costfunction $f, \sum_{i=1}^{n} f\left(s_{i}\right)=f\left(\bigcup_{i=1}^{n} s_{i}\right)$ holds.

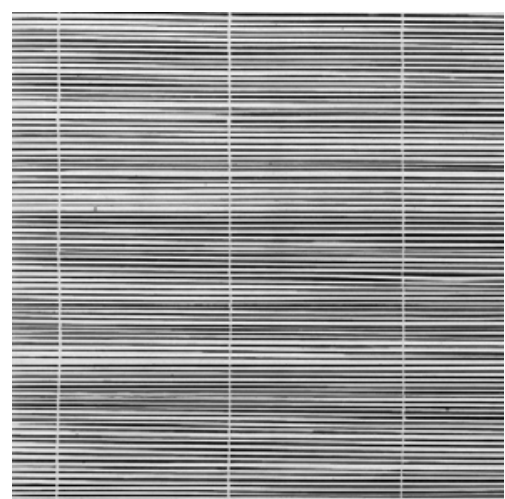

(a) Brodatz texture D49

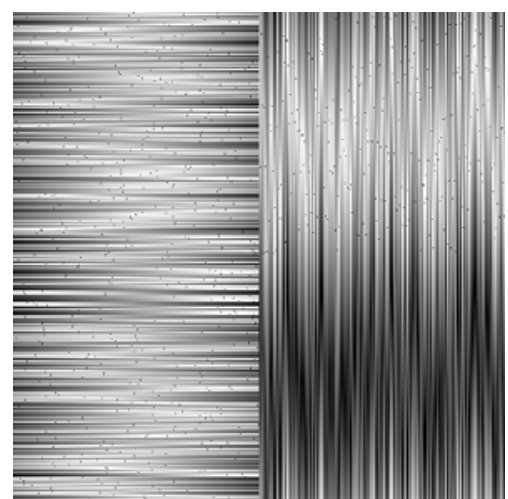

(b) Artificial test image

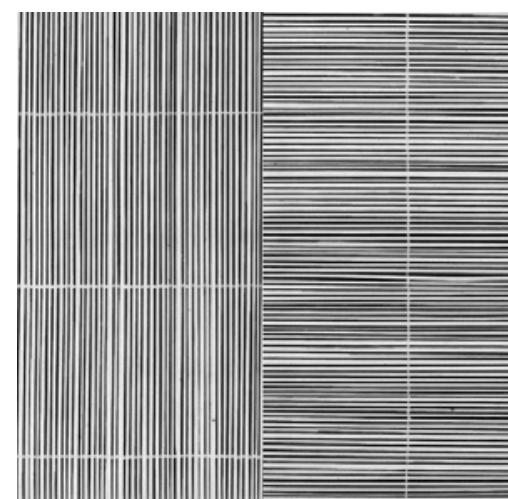

(c) Brodatz texture test image

Fig. 2 Test images, $512 \times 512$ pixels, 8bpp.

In a first step we aim at verifying the superiority of the WP-transform over classical wavelet transforms for textured images. A couple of artificially generated images with strong high frequency components in a singular direction (see the two halves of Figure 2.b) are tested for their suitability for WP-coding. All the wavelet-packet decompositions that any of the used costfunctions produce for these pictures outperform the pyramidal decomposition by at least $4 \mathrm{db}$. The test results for the set of tested natural images, consisting of Brodatz-texture images [9] (Figure 2.a) and textile fabric images, are similar to the artificial images - for fine, textured images, the wavelet-packet decomposition outperforms the pyramidal decomposition

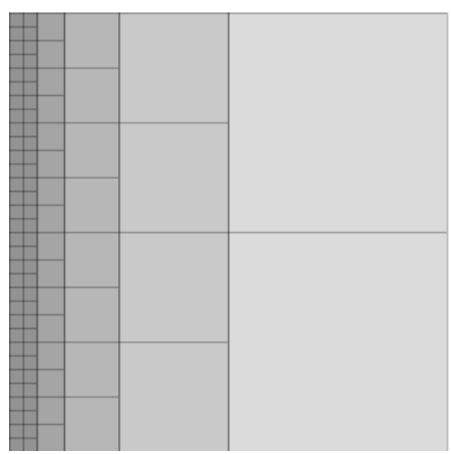

Fig. 3 WP-Structure of D49 significantly (Figure 4.a). It is interesting to observe that the performance of the different costfunctions varies from image to image. None of the used costfunctions turned out to return the best decomposition with respect to PSNR in any case. Also, there seems to be no direct correlation between the value of the costfunction and the PSNR of the reconstructed image.

In the second step we test the adaptive object-based approach and create hybrid images containing two rectangular objects of the same size, which are chosen from the set of the previously tested images with the subband structures being as different as possible. By limiting the shape of the objects to rectangles, some of the special issues that come with arbitrary shapes [6] are not relevant for this setup. In our implementation the bitbudget is allocated proportionally to the size of the object, i.e. as both objects in the setup have the same size, they are both granted the same bitbudget.

As can be clearly seen from Figure 4.b, for the artificial test image the object-based adaptation approach outperforms the global adaptation significantly across the entire bitrange considered. Additionally, the non-adaptive classical pyramidal decomposition is clearly inferior to the results achieved by adaptation for this class of images. In Figure 4.c, it can be seen that for the hybrid Brodatz test image, the object-based adaptation is superior for 


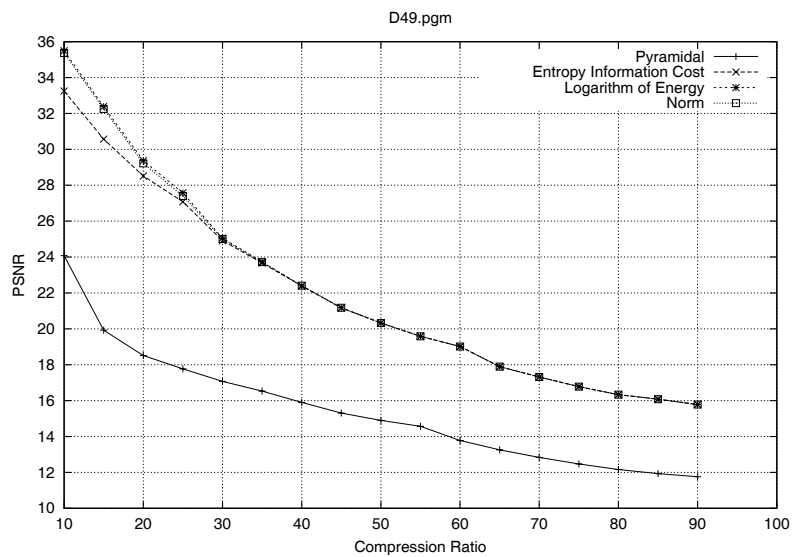

(a) Results for Figure 2.a

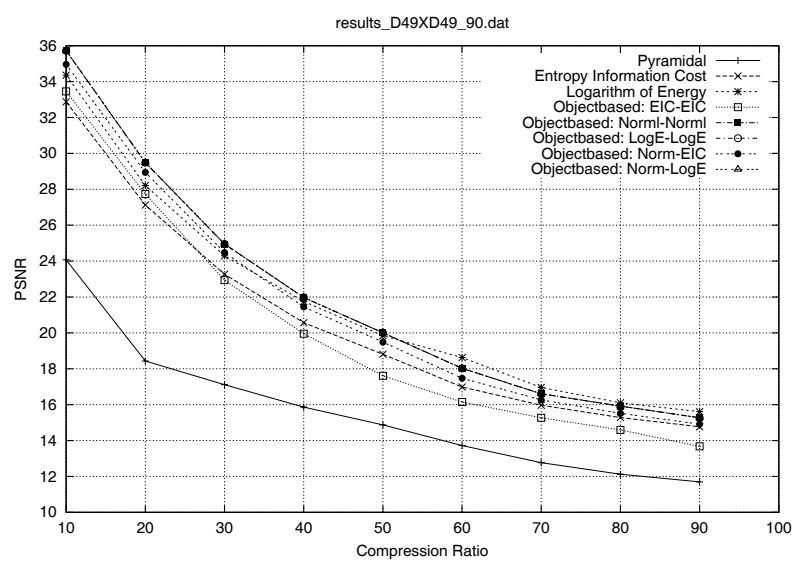

(c) Results for Figure 2.c

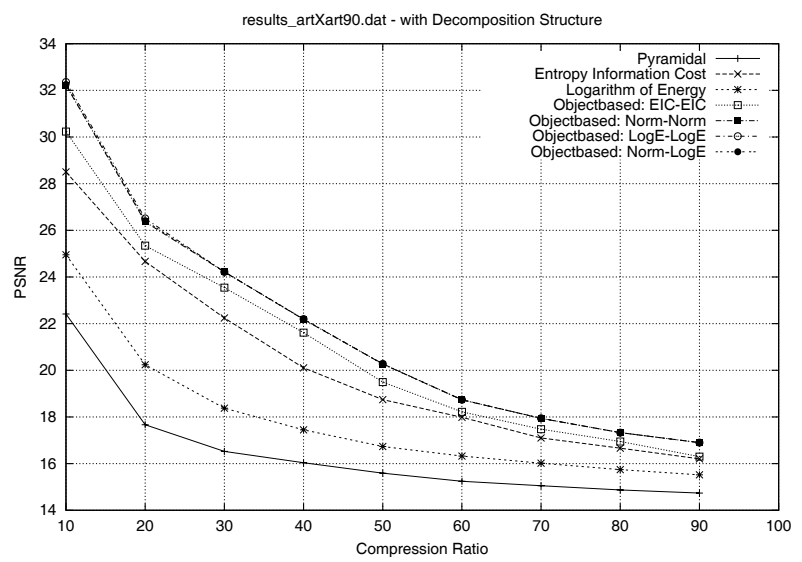

(b) Results for Figure 2.b

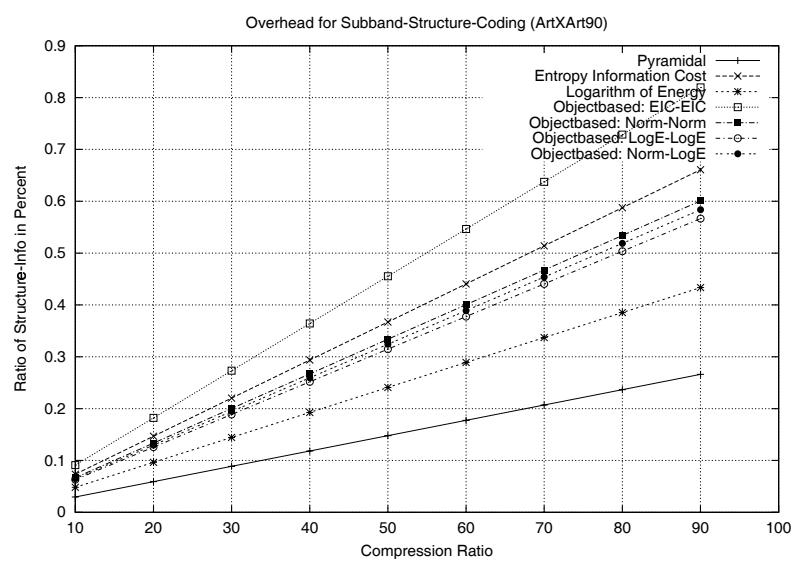

(d) Overhead for coding the WP-Structures

Fig. 4 Test results

compression-ratios up to 50. With higher compression global adaption gains an advantage, because, due to the limited number of coded coefficients, each coefficients contains information about all objects. Generally, the more dissimilar the subband structures of the various objects are, the higher is the compression ratio up to which local adaption outperforms global adaption.

One might argue that there is considerable overhead associated when storing the WP subband structure for each object separately. But the quadtree representation of the subband structures is very efficient - Figure 4.d shows that even in the case of high compression ratios this overhead never exceeds $1 \%$ of the entire bitbudget of the corresponding objects.

\section{CONCLUSION}

When dealing with images containing objects of heterogenous traits in frequency space, the adaptive object-based approach produces better compression results than non-object-based and non-adaptive approaches. Our approach proved to be suitable for a specific class of images, yet it has one drawback. Since the allocation of the bitbudget for an object is determined by the size of the object only, we obtain good results for images composed of objects showing similar levels of entropy or visual energy only.

For images containing very different objects in the aforementioned sense, the suboptimal 
bitbudget allocation strategy does not allow the object-based approach to outperform the global optimization. In future work, we will address this issue by using a more sophisticated strategy for bitbudget allocation based on size and features of the different objects.

\section{REFERENCES}

[1] R. Coifman and M. Wickerhauser, Entropy based methods for best basis selection, IEEE Transactions on Information Theory, vol. 38, no. 2, pp. 719-746, 1992.

[2] M. Wickerhauser, Adapted wavelet analysis from theory to software. Wellesley, Mass.: A.K. Peters, 1994.

[3] F. G. Meyer, A. Z. Averbuch, and J.-O. Strömberg, Fast adaptive wavelet packet image compression, IEEE Trans. on Image Process., vol. 9, pp. 792-800, May 2000.

[4] C. Herley, J. Kovacevic, K. Ramchandran, and M. Vetterli, Tilings of the time-frequency plane: Construction of arbitrary orthogonal bases and fast tiling algorithms, IEEE Trans. on Signal Process., vol. 12, no. 41, pp. 3341-3359, 1993.

[5] N. M. Rajpoot, R. G. Wilson, F. G. Meyer, and R. R. Coifman, A new basis selection paradigm for wavelet packet image coding, in Proceedings of the IEEE International Conference on Image Processing (ICIP'01), (Thessaloniki, Greece), pp. 816-819, Oct. 2001.

[6] S. Li and W. Li, Shape-adaptive discrete wavelet transform for arbitrarily shaped visual object coding, IEEE Transactions on Circuits and Systems for Video Technology , vol. 10, pp. 725-743, Aug. 2000.

[7] R. Kutil, A significance map based adaptive wavelet zerotree codec (SMAWZ), in Media Processors 2002 (S. Panchanathan, V. Bove, and S. Sudharsanan, eds.), vol. 4674 of SPIE Proceedings, pp. 61-71, Jan. 2002.

[8] A. Said and W. A. Pearlman, A new, fast, and efficient image codec based on set partitioning in hierarchical trees, IEEE Transactions on Circuits and Systems for Video Technology, vol. 6, pp. 243-249, June 1996.

[9] P. Brodatz, Textures: A Photographic Album for Artists and Designers. New York: Dover Publications, 1966. Pictures downloaded from http://www.ux.his.no/ tranden/brodatz.html (Trygve Randen). 